\title{
Médiévales
}

Langues, Textes, Histoire

54 | printemps 2008

Frères et sœurs

\section{Un avis retrouvé de Jacques Fournier}

A lost tract of Jacques Fournier re-discovered

\section{Sylvain Piron}

\section{(2) OpenEdition}

Journals

Édition électronique

URL : https://journals.openedition.org/medievales/5062

DOI : $10.4000 /$ medievales. 5062

ISSN : $1777-5892$

\section{Éditeur}

Presses universitaires de Vincennes

\section{Édition imprimée}

Date de publication : 1 juin 2008

Pagination : 113-134

ISBN : 978-2-84292-217-7

ISSN : 0751-2708

\section{Référence électronique}

Sylvain Piron, « Un avis retrouvé de Jacques Fournier », Médiévales [En ligne], 54 I printemps 2008, mis en ligne le 10 septembre 2010, consulté le 22 avril 2022. URL : http://journals.openedition.org/ medievales/5062 ; DOI : https://doi.org/10.4000/medievales.5062

Ce document a été généré automatiquement le 22 avril 2022

Tous droits réservés 


\title{
Un avis retrouvé de Jacques Fournier
}

\author{
A lost tract of Jacques Fournier re-discovered
}

Sylvain Piron

1 La postérité a été injuste avec Jacques Fournier, pape sous le nom de Benoît XII. Au cours des dernières décennies, l'attention des historiens s'est concentrée presque exclusivement sur le fameux registre d'inquisition de l'évêque de Pamiers, qui n'est au fond qu'un document de sa pratique administrative ${ }^{1}$. Dans le même temps, les travaux savants du théologien cistercien sont demeurés dans l'oubli le plus profond. Rien ou presque ne subsiste de sa production universitaire ${ }^{2}$. C'est à Avignon, durant son cardinalat et son pontificat, qu'il a composé sa grande œuvre théologique, mais ce monumental commentaire sur l'évangile de Matthieu, luxueusement conservé en six volumes dans la bibliothèque pontificale, n'a guère rencontré d'écho. Il n'a été publié qu'en partie et accidentellement, par un dominicain qui pensait éditer les œuvres de son confrère Benoit $\mathrm{XI}^{3}$. Un autre aspect de sa production intellectuelle est lié à sa fonction de conseiller théologique de Jean XXII dans les procès doctrinaux les plus importants des années $1325-1333^{4}$. De cette activité, qui est à la fois l'une des causes et le signe de son ascension fulgurante à la curie pontificale dans cette période, seul le dossier de ses interventions dans le débat sur la vision béatifique a été conservé, mais il demeure encore pour l'essentiel inédit ${ }^{5}$. Dans les pages qui suivent, je voudrais ajouter un nouveau document à cette liste, en identifiant l'une de ses plus anciennes expertises théologiques, rendue à l'occasion de la dernière phase du procès contre le commentaire de l'Apocalypse du théologien franciscain Pierre de Jean Olivi ${ }^{6}$.

Initié en 1318, dans la foulée de la condamnation au bûcher de quatre franciscains Spirituels, puis mis en sommeil pendant quelques années, ce procès fut relancé après novembre 1324, lorsque Jean XXII procéda lui-même à une lecture du texte incriminé et soumit quatre (ou plutôt cinq) nouveaux article à l'examen de quelques experts. On connaît les réponses données par l'évêque de Florence, Francesco Silvestri, et par l'avocat franciscain Bonagrazia de Bergame; ces textes répliquent également à une ultime défense de l'orthodoxie d'olivi présentée par Ubertin de Casale au sujet des 
mêmes articles ${ }^{7}$. À ces documents, il convient d'ajouter un avis anonyme, contenu dans un manuscrit de la bibliothèque municipale d'Avignon, répertorié depuis longtemps et pourtant jusqu'à présent négligés. Bien qu'il ne soit lui aussi que partiel, ce texte constitue de loin la discussion la plus approfondie des thèses d'olivi dans cette dernière phase du procès et cette contribution a probablement joué un rôle majeur dans la préparation de la sentence, rendue en février 1326. C'est cette œuvre qui semble devoir être attribuée à Jacques Fournier9.

Description du texte

3 Le codex 1087, anciennement conservé à la bibliothèque des Célestins d'Avignon, est un recueil de pièces diverses réunies à une date tardive, les premières étant de nature juridique ou politique. L'avis anonyme qui occupe les derniers cahiers du volume est précédé du Tractatus de antichristo du dominicain Jean Quidort, les deux pièces étant copiées dans la seconde moitié du XIV siècle par des mains différentes. Le connaisseur qui a réuni ces textes a ainsi associé deux des critiques les plus précises des attentes joachimites d'une crise apocalyptique, destinée à précéder la venue d'un troisième âge de l'Église. Le catalogue dressé par L.-H. Labande indique la présence, dans la dernière partie du codex, de deux textes distincts qui auraient visé, à des titres divers, la Lectura super Apocalipsim : un "Traité contre la doctrine de l'abbé Joachim et de Pierre-Jean d'Olivi, exprimée dans leurs commentaires de l'Apocalypse", aux folios 220r-242r, suivi, aux folios $242 \mathrm{v}-275 \mathrm{r}$, d'un « Traité adressé au pape sur le rapport des inquisiteurs chargés par Jean XXII d'examiner la doctrine " du même Olivi ${ }^{10}$. Ces deux traités forment en réalité deux parties d'une même œuvre, transmise de façon très incomplète. On les présentera l'une après l'autre, avant de chercher à en identifier l'auteur.

Première partie de l'avis : sur l'intelligence spirituelle du troisième âge

4 Le premier texte débute de façon abrupte, au milieu d'une phrase qui appartient au dixième chapitre de la Lectura super Apocalipsim ${ }^{11}$. Le fait que ces mots soient présentés comme l'incipit du texte et précédés d'un pied de mouche signifie que le copiste avait devant lui un texte acéphale, qui avait été privé d'un ou plusieurs folios initiaux. Cette citation est elle-même suivie d'un montage de deux extraits du dix-neuvième chapitre de la Lectura, décrivant les débuts du troisième âge après la destruction de Babylone. Il faut donc supposer qu'une chaîne plus longue de citations du même ouvrage occupait les premières pages manquantes et qu'elle était elle-même précédée d'un exposé général de la question soumise à examen.

5 Devaient également figurer dans ces pages perdues des extraits de Joachim de Fiore ainsi qu'une justification du rapprochement opéré entre les interprétations de l'Apocalypse des deux théologiens. En effet, la première remarque qui suit les citations d'olivi souligne l'incertitude du moment marquant le début des sixième et septième périodes de l'Église dans lequel devrait avoir lieu la nouvelle infusion de l'Esprit Saint «dont il est fait mention dans les positions de Joachim et de Pierre Jean citées plus haut ${ }^{12}$ ». Cette incise permet de présenter ensuite, tour à tour, les opinions contradictoires des deux auteurs, aussi bien quant à la date de cette nouvelle infusion qu'à propos de la chronologie des sept états de l'Église, au moyen d'une nouvelle série d'extraits de leurs commentaires respectifs de l'Apocalypse.

6 Ce n'est qu'un peu plus loin que l'on comprend l'objet précis de cet examen. Il ne s'agit ici, écrit l'auteur de l'avis, que de répondre à une question concernant un accroissement de l'intelligence spirituelle au début du sixième état de l'Église - 
question qui correspond littéralement au deuxième des articles extraits par Jean XXII ${ }^{13}$. L'auteur de cet avis ne s'est donc pas contenté de répondre à la question posée. Très intelligemment, il a choisi lui-même de confronter les positions d'olivi à celles de son principal inspirateur. L'un des éléments qu'il en retire, dans la première partie de sa réponse, lui permet de souligner les discordances entre leurs chronologies des temps futurs. Pour Joachim, le troisième âge devait débuter avec saint Benoît, tandis que pour le frère mineur, c'est saint François qui fournit le premier repère. À deux reprises, l'auteur de l'avis ne manque pas de souligner que ces prédictions sont déjà démenties par les faits, en révélant ainsi la date à laquelle il écrit. Selon les calculs de Joachim, le troisième âge aurait commencé depuis huit siècles s'il faut partir de l'époque de saint Benoît, depuis 139 ans s'il faut compter à partir de la rencontre entre Joachim et Urbain III, ou 125 ans si la date fatidique est l'an 1200 - ces trois différentes dates étant en effet alternativement mentionnées par Joachim ${ }^{14}$. De même, depuis que la Règle franciscaine a été reconnue et « canonisée » en 1223 , cent deux ans se sont déjà écoulés ${ }^{15}$.

7 Dans le même sens, l'auteur cite un passage d'un commentaire sur Daniel qu'il attribue à Olivi mais qui est en réalité l'œuvre de son disciple, Barthélemy Sicard ${ }^{16}$. Ce texte, bien connu des Spirituels et des béguins, n'a, à ma connaissance, jamais été employé par leurs adversaires ni été attribué à leur "saint père »; il n'est mentionné ni par Raymond de Fronsac, ni par Bonagrazia de Bergame, successivement procureurs de l'ordre franciscain et de ce fait aux premières loges du combat contre la dissidence languedocienne; son utilisation dénote ainsi une connaissance approfondie des courants spirituels franciscains du Midi. Rédigeant son commentaire dans la première décennie du XIV siècle, Barthélemy présentait un calcul explicite de la date à laquelle serait détruit l'Antéchrist : en comptant comme des années les 1290 jours mentionnés au dixième chapitre du livre de Daniel, et en faisant partir cette durée de la passion du Christ, on parvient à l'an 1324 de l'incarnation dans lequel devrait s'achever la grande tribulation. C'est sans doute en pensant à cette échéance que l'hérésiarque béguine Prous Boneta, dans sa confession donnée devant l'inquisition lors de l'été 1325, plaçait la fin de l'Église romaine à la Noël 1323, en associant cet évènement à la bulle Cum inter nonnullos, comprise comme équivalant à la condamnation finale de la Lectura super Apocalipsim $^{17}$. L'auteur de l'avis ne se prive pas de souligner que cette prédiction a été démentie par les faits puisqu'il écrit en l'année 1325 de l'Incarnation et que, loin d'être détruite, l'Église romaine est au sommet de sa dignitée ${ }^{18}$.

8 L'inanité des annonces de Joachim et de Pierre suffit à démontrer qu'ils sont l'un et l'autre de faux prophètes ${ }^{19}$. Les pages suivantes réfutent leur présomption d'avoir voulu prédire les temps futurs en s'appuyant principalement sur de nombreuses citations de saint Augustin. Sans que le manuscrit marque précisément les articulations du texte, on comprend qu'est ensuite examinée une autre erreur commise par les deux auteurs qui qualifient l'Église romaine de Babylone, devant être détruite avant la mort de l'Antéchrist ${ }^{20}$. Augustin est à nouveau la principale autorité sollicitée pour montrer que Jérusalem et Babylone représentent les deux cités des réprouvés et des élus qui subsisteront entremêlées jusqu'à la fin des temps. Le découpage temporel de l'histoire de l'Église en sept périodes est lui-même mis en cause puisque la seule distinction acceptable est celle qui reconnaît un temps avant la loi, un autre sous la loi et un troisième sous la grâce, lequel doit durer de l'incarnation du Christ jusqu'au jugement dernier sans admettre d'autre subdivision en son sein. 
9 Ces premières critiques ne constituent qu'un préambule à l'examen de la question posée, destiné à rappeler que les présupposés de l'affirmation suspecte sont eux-mêmes faux. Toutefois, dans l'hypothèse où ils seraient acceptables ${ }^{21}$, l'interrogation porte sur l'éventuel surcroît d'intelligence spirituelle qui devrait se produire à ce moment, que le Christ aurait promis en Jean 16,13 (Cum venerit ille spiritus...). Ces deux points sont traités séparément. L'auteur s'interroge d'abord sur le sens de la tournure inhabituelle employée par Olivi qui parle d'une "expérience gustative et tangible» (gustativa et palpativa experiencia): ces mots lui paraissent désigner métaphoriquement une connaissance intellectuelle de Dieu, inspirés des images utilisées dans le Psaume 33,9 et en Jérémie 15,16 ou 20,9 où les paroles du Seigneur sont comme "goûtées » et «touchées $»^{22}$. L'expression peut être comprise de plusieurs façons. Puisque la citation examinée ajoute qu'une telle expérience doit faire connaître «toute la sagesse du Verbe incarné et la puissance de Dieu le Père ", il doit nécessairement s'agir d'une connaissance pleine et parfaite de Dieu, occupant tout l'intellect humain, qui ne fait voir que Dieu en toutes les créatures ${ }^{23}$. Déployant une à une les conséquences d'une telle vision béatifique donnée dans cette vie terrestre, l'auteur montre qu'elle reviendrait à placer les humains dans un état de raptus continuel ; ceux-ci pourraient se passer de l'Église et des sacrements qui ne sont nécessaires que tant que Dieu n'est pas vu mais seulement cru par la foi ; une telle vision béatifiante rendrait ses bénéficiaires incapables de commettre le moindre péché (inpeccabilis). On peut donc opposer à cette opinion la bulle Ad nostrum qui desideranter prise lors du Concile de Vienne pour condamner les adhérents d'une supposée secte du libre esprit que leur divinisation en cette vie aurait placés au-dessus de la condition des fidèles ${ }^{24}$. Un tel rapprochement entre les béguins du Rhin et ceux du Midi n'avait jamais été effectué dans les documents connus à ce jour. En 1325, la dissidence radicale du groupe mené par Prous Boneta l'avait rendu particulièrement pertinent.

Cette nouvelle connaissance peut également être prise au sens où l'entend Joachim, d'un éclaircissement de toutes les figures et obscurités de l'Écriture. Une telle perspective reçoit à son tour une longue réfutation qui porte cette fois sur le principe même d'un accroissement de l'intelligence spirituelle au cours de l'histoire, grâce à laquelle le message du Christ pourrait être mieux compris que les apôtres ne l'ont fait ${ }^{25}$. C'est dans ce cadre qu'est discutée l'annonce de la venue du Paraclet faite dans le seizième chapitre de l'Évangile de Jean ; pour l'auteur, cette annonce s'est totalement accomplie dans la Pentecôte; il serait insensé, hérétique et présomptueux de prétendre avoir compris ce que les apôtres n'étaient pas capables d'entendre ${ }^{26 .}$

11 L'avis entreprend ensuite de répondre à des arguments avancés pour « excuser » les paroles d'olivi. On retrouve ici, sous une forme sensiblement abrégée, les mêmes « excuses » auxquelles répond Bonagrazia de Bergame dans les Allegationes ${ }^{27}$. Ubertin de Casale est assurément le seul personnage présent à la curie en 1325 qui pouvait avoir le cran de défendre encore la Lectura super Apocalipsim; il l'a fait en cherchant une nouvelle fois à minimiser la gravité de certains propos : la nouvelle infusion de l'Esprit saint doit se comprendre comme «l'un des multiples effets de Dieu lors de l'approche de la fin des temps »; elle n'est que de l'ordre d'une grâce spéciale, gratis data, et non pas une grâce accordant à ses bénéficiaires une quelconque supériorité sur les saints des siècles passés dans l'ordre de la charité. Quant à la nouvelle connaissance «tangible» des secrets divins, elle ne concernerait que la vision béatifique des bienheureux qui constitue la septième période de l'Église et qui, unie à la sixième, 
constitue le troisième temps de l'histoire de la création. La réplique n'a aucun mal à montrer que la nouvelle intelligence spirituelle concerne déjà la sixième période et que Joachim et Olivi « placent les humains du troisième âge au-dessus des apôtres » quant à la clarté de leur connaissance de Dieu ${ }^{28}$.

Seconde partie de l'avis : sur les articles des maîtres

Le dernier document contenu dans le manuscrit, décrit dans le catalogue de Labande comme une œuvre distincte, est étroitement lié au précédent. Copié de la même main, il s'agit à nouveau d'un examen d'articles extraits de la Lectura, mené par le même auteur $^{29}$. Ce texte débute par une préface adressée au pape, rappelant le rôle de ce dernier comme défenseur de la foi; il mentionne ensuite l'apparition récente d'une doctrine erronée qui a déjà été réprouvée par de nombreux docteurs, faisant ainsi allusion à la Littera magistrorum produite en 1319 par une commission formée de huit maitres en théologie ${ }^{30}$. Répondant à une demande du souverain pontife, et écrivant sous sa correction, l'auteur annonce qu'il présentera d'abord certains des articles réputés suspects par les maîtres avant d'exposer son propre jugement puis de répondre aux excuses qui prétendent que la "postille» ne contient rien d'hérétique ${ }^{31}$. Cette brève introduction ne concerne donc pas les articles choisis par le pape, mais uniquement ceux de la Littera magistrorum; elle ne constitue pas une préface générale à l'avis rendu à Jean XXII, mais une simple présentation de sa deuxième partie.

13 En effet, si une rubrique annonce ensuite un articulus primus, il ne s'agit pas d'une discussion du premier article examiné par Bonagrazia et Francesco Silvestri, mais du premier article de la Littera magistrorum. Toutefois, les citations des premières pages du prologue de la Lectura données dans ce cadre sont plus prolixes et incorporent également l'extrait retenu par les maîtres au titre de leur deuxième article ${ }^{32}$. L'essentiel de la réfutation qui suit se concentre d'ailleurs sur ce dernier passage dans lequel Olivi traite de la « prééminence notable » du sixième état de l'Église sur les cinq premiers et le présente comme «le début d'un nouveau siècle évacuant d'une certaine façon le siècle précédent » qui ferait revenir au premier état de l'Église, comme si l'histoire de celle-ci décrivait un cercle ${ }^{33}$. À nouveau, chacune des assertions contenues dans la citation est passée au crible, l'une après l'autre. La prééminence du sixième état impliquerait notamment qu'il serait supérieur, non seulement à celui des apôtres mais également à celui du Christ, puisque ce dernier ne peut être exclu du premier état de l'Église dont il est le fondateur ${ }^{34}$. Le thème d'une "évacuation du siècle ancien ", pris à la lettre, peut signifier que l'Évangile et les sacrements de la nouvelle loi seront abandonnés avant la fin des temps. L'auteur de l'avis suggère qu'olivi a trouvé son inspiration dans des passages de la Concordia de Joachim, longuement reproduits ici, mais il ne fait pas d'allusion à la condamnation sur ce thème de l'évangile éternel de Gérard de Borgo San Donnino ${ }^{35}$. En comprenant le "siècle ancien" au sens de la "vétusté du péché », on tomberait dans l'erreur des Pélagiens ${ }^{36}$. L'« évacuation » peut encore se comprendre comme un retour à la pauvreté de l'Église primitive qui n'aurait eu qu'un « simple usage de fait » des biens matériels ${ }^{37}$. Étonnamment, l'auteur ne prend pas appui sur la bulle Cum inter nonnullos pour dénoncer cette référence à la pauvreté du Christ et des apôtres, comme le fait Bonagrazia dans les Allegationes ${ }^{38}$; il se fonde plutôt sur des arguments exégétiques pour montrer qu'un tel retour à la pauvreté primitive, quant au nombre des apôtres ou à leur mode de vie, n'est fondé nulle part dans l'Écriture ${ }^{39}$. 

l'Église, permet de prolonger cette critique. L'ordre franciscain ne constitue qu'une petite part du peuple chrétien et sa particularité quant à la pauvreté ne rend en rien ses membres plus vertueux que les martyrs, confesseurs et docteurs des temps passés; chaque ordre religieux est à sa façon conforme au Christ, aucun ne l'est totalement et les frères mineurs ne peuvent revendiquer ni exclusive ni supériorité à cet égard ${ }^{40}$. Le modèle de l'histoire de l'Église ne peut être un cercle mais une ligne droite qui n'admet aucune flexion. L'idée même d'une «rénovation de la vie évangélique » qui aurait été effectuée par saint François est repoussée : la vie évangélique, depuis sa fondation, n'a pas vieilli puisqu'elle se poursuit constamment en ses fidèles depuis la fondation de l'Église ${ }^{41}$. Il faudrait être insensé pour affirmer que quiconque professe la Règle de saint François imite davantage le Christ que les saints les plus vénérés par l'Église ${ }^{42}$. La « rénovation » dont parle saint Paul dans l'Épître aux Éphésiens en appelant à "se dépouiller du vieil homme » ne concerne en rien l'abdication des richesses ou quelque autre vœu monastique, mais «la justice et la sainteté qui vient de la vérité " et l'absence de tout vice ${ }^{43}$. Elle s'accomplit principalement par la pratique vertueuse et l'évitement des péchés et non pas au moyen d'un vœu ${ }^{44}$. De la même façon, l'idée que l'Église devrait être réédifiée n'a pas de sens puisqu'elle n'a jamais été détruite ${ }^{45}$. Et rien, dans l'Écriture, ne suggère qu'elle devrait se réduire, lors des persécutions de l'antéchrist, à un nombre de fidèles aussi restreint que celui de l'Église primitive ${ }^{46}$. Quant à placer la milice future du Christ sous le signe de saint François, au nom de ses stigmates qui l'auraient "signé du signe du Christ », cette thèse impliquerait que tous les élus appelés à lutter contre l'antéchrist devraient être instruits par des frères mineurs, à l'exclusion de tout autre ordre religieux ${ }^{47}$. Les excuses présentées par Ubertin, protestant qu'Olivi a voulu conserver une unité de l'Église romaine universelle et non pas poser deux Églises successives ou que l'expression "Église charnelle » ne désigne pas l'Église romaine mais la seule cohorte des réprouvés, donnent l'occasion d'un redoublement des précédentes critiques ${ }^{48}$.

ne fois achevé cet article, un paragraphe de transition annonce la section suivante. Le thème d'une supériorité des deux derniers états de l'Église est davantage argumenté dans le septième notabile du prologue de la Lectura - septième notabile dont la seule annonce dans les premières pages du prologue avait formé la matière du deuxième article de la Littera magistrorum. C'est également dans ce notabile qu'a été pris l'extrait pointé dans le quatrième article; or, puisque la matière des deuxième et troisième articles a été épuisée, c'est à ce quatrième point que l'on vient maintenant; pour l'introduire, le septième notabile est reproduit en totalité ${ }^{49}$. Sans entrer dans tous les détails de ces derniers folios, qui reprennent souvent, en les approfondissant, des thèmes abordés dans les pages précédentes, on peut au moins en retenir un intéressant argument de méthode. L'auteur reproche à Olivi et Joachim, toujours critiqués de conserve, d'avoir voulu tirer leurs arguments de la «théologie mystique » alors que celle-ci n'a pas de valeur démonstrative (argumentabilis non est). Ce type de démarche les a incité à rapprocher toutes les occurrences du nombre six qu'ils ont trouvé dans l'Écriture des six états de l'Église, sans tenir compte du sens littéral ni du contexte particulier de ces différents $" s i x »^{50}$.

Structure d'ensemble

Au terme de ce tour d'horizon, il est possible de dégager la structure d'ensemble de l'œuvre plus vaste dont proviennent ces fragments, en s'appuyant sur la présence de 
plusieurs renvois internes. Le premier document est décrit comme une "question ${ }^{51}$; elle contient elle-même une référence à la "question précédente » et à ce qui sera dit plus loin ${ }^{52}$. Cette désignation correspond à la forme de la consultation demandée par Jean XXII, qui interrogeait les experts sollicités sur l'orthodoxie de brefs passages de la Lectura qu'il avait lui-même épinglés ${ }^{53}$. Dans ce cadre, le premier texte du manuscrit d'Avignon correspond à la deuxième question de cette consultation. Les deux textes suivants sont quant à eux désignés comme des « articles » et leur numérotation, comme on l'a vu, correspond à celle de la Littera magistrorum. Le premier article contient des renvois à la première et à la deuxième question ${ }^{54}$. Le traitement du quatrième article fait référence au précédent et à la deuxième question ${ }^{55}$.

17 La seule certitude que l'on puisse retirer de ces indications concerne l'existence d'une première question, correspondant au premier article choisi par Jean XXII, dans laquelle l'auteur de l'avis aurait notamment démontré que saint Paul a exercé un droit de propriété sur les biens temporels ${ }^{56}$. Si l'on s'est étonné de ne pas trouver davantage de discussion sur la pauvreté du Christ et des apôtres, c'est que le point avait dû être largement abordé dans ce cadre. Rien, en revanche, ne permet de savoir si les deux questions suivantes auxquelles a répondu Francesco Silvestri ont également été traitées par l'auteur de l'avis, de même qu'il est impossible de savoir s'il a poursuivi son commentaire des articles de la Littera magistrorum au-delà du quatrième. En effet, le rassemblement opéré dans le manuscrit d'Avignon concerne principalement les problèmes posés, chez Joachim et Olivi, par le progrès spirituel du troisième âge. Le voisinage du traité De antichristo de Jean Quidort pourrait laisser penser que cette sélection a été intentionnelle, et qu'elle aurait de ce fait négligé l'examen des trois autres questions posées par le pape qui touchent moins directement cet aspect de l'eschatologie joachimite.

Pour fragmentaire qu'il soit, ce long avis anonyme apporte un dernier enseignement de grande importance. Lors de cette seconde consultation sur la Lectura, en 1325, Jean XXII n'a pas simplement substitué au premier rapport de 1319 le résultat de sa propre lecture. Les historiens se sont souvent étonnés de constater que le pape avait choisi des points apparemment mineurs, en négligeant des passages plus choquants qu'avaient déjà fortement soulignés les huit maîtres ${ }^{57}$. La raison en est que son intervention n'a pas annulé le premier rapport mais a plutôt cherché à le compléter. Pour des raisons évidentes, les experts consultés ont donné par priorité leur avis sur les nouvelles questions soulevées par le souverain pontife; toutefois, tant Bonagrazia de Bergame (à ce qu'il annonce au début de ses Allegationes $\left.{ }^{58}\right)$ que l'anonyme du manuscrit d'Avignon ont ensuite entrepris de discuter certains articles de la Littera. Si ce résultat permet de clarifier le sens de cette dernière étape de la procédure, il rend de plus en plus hasardeuse toute conjecture quant au contenu de la sentence finale, rendue en février 1326, dont le texte n'a pas été conservé.

Identification de l'auteur : Jacques Fournier?

Bien qu'il ne soit transmis que très partiellement, cet avis anonyme est nettement plus long que les deux autres rapports connus et traite les sujets abordés avec une ampleur de vue bien supérieure. Il est d'autant plus tentant de chercher à identifier son auteur que peu de candidats sont sur les rangs. À ce jour, ne sont attestés que deux avis remis au pape en cette occasion et non encore retrouvés. Comme l'a montré Patrick Nold, une réponse de Bertrand de la Tour «contre certains arguments qui semblent excuser de toute hérésie la doctrine de Pierre Jean", autrefois conservée dans un manuscrit 
d'Assise à présent perdu, ne doit pas être associée aux débats des années 1310-1311 comme on le pensait auparavant, mais devrait plutôt être rapportée à cette ultime phase de la procédure contre la Lectura ${ }^{59}$. De fait, la tournure employée est très proche de celle utilisée par Bonagrazia et l'anonyme pour désigner les « excuses » d'Ubertin. Cela signale que Bertrand, qui avait déjà donné son avis sur la Lectura en tant que membre de la commission de 1319, a seulement été requis par Jean XXII de répondre à cette ultime défense. Il ne saurait donc être l'auteur de l'avis du manuscrit d'Avignon qui prend en considération les deux séries d'extraits. Les Allegationes de Bonagrazia font pour leur part allusion à une intervention du cardinal de Sainte-Sabine, Guillaume de Peyre Godin, mais cette référence vise sans doute son rôle comme juge délégué du procès intenté contre Ubertin en 1324. Il ne reste donc en lice qu'un seul candidat sérieux, le successeur même de Jean XXII.

Dans son article consacré aux avis théologiques rendus par Jacques Fournier ${ }^{60}$, Josef Koch a mis à profit des fragments de ces documents perdus cités par des auteurs ultérieurs, notamment par le théologien augustinien Johannes Hiltalingen de Bâle. Comme l'a noté Damasus Trapp, l'une des particularités «modernes » d'Hiltalingen est le soin qu'il apporte à citer précisément ses sources ${ }^{61}$. La troisième de ses Responsiones, soutenues alors qu'il était bachelier formé à Paris en 1368, traite du caractère méritoire de la pauvreté volontaire. C'est à ce propos qu'il utilise les avis rendus par Benoît XII contre Michel de Césène et contre Olivi. Les citations de ce dernier texte concernent le premier article de la consultation, ce qui ne permet pas de mener une confrontation directe avec le manuscrit d'Avignon dans lequel cette question manque. Dans une première allusion, Hiltalingen cite avec approbation les arguments avancés par Jacques Fournier, dans le troisième chapitre de cette première question, pour montrer que, selon l'Évangile et le droit canon, il est permis aux évêques de posséder des biens ${ }^{62}$. En revanche, un peu plus loin, l'autorité de Benoît XII est traitée avec moins de ménagements.

21 Le deuxième corollaire tiré par le théologien bâlois s'inspire de la formulation même du premier article extrait de la Lectura: si le pontificat du Christ a d'abord été donné en Pierre à la lignée de la vie évangélique, purifiée par la pauvreté, il ne semble pas s'ensuivre formellement que le souverain pontife y soit maintenant obligé63. Sans connaître la position d'Olivi autrement qu'à travers sa critique par Jacques Fournier, Hiltalingen ne retient en réalité que la moitié de son raisonnement : en dissociant les statuts du Christ et de son vicaire, il est possible d'accepter simultanément la pauvreté du Christ et des apôtres et de reconnaître que le pontificat suprême n'est pas tenu d'agir selon ce modèle; pour cette raison, "il apparaît que tout le procès mené par Benoît XII contre Pierre Jean dans le premier article, chapitre 13 et ultime, n'est que très peu ou pas du tout concluant, bien que Pierre doive être supposé être hérétique, condamné par l'Église, comme le montre Benoît au même lieu ${ }^{64}$ ».

Les lignes suivantes reproduisent un long extrait de l'avis de Jacques Fournier, présentant les divers sens dans lesquels peut être compris l'expression «vie évangélique ${ }^{65} "$, ainsi qu'une remarque attribuée aux expositores de Pierre - le terme étant évidemment à corriger par excusatores. Dans la mesure où les problèmes traités ne se recouvrent pas, aucune conclusion certaine ne peut être tirée d'un rapprochement entre ces citations et l'avis anonyme. On notera toutefois une similitude dans la démarche engagée. Comme l'auteur de l'avis d'Avignon, Jacques Fournier semble lui aussi avoir pris un par un chaque élément de la citation soumise à examen ${ }^{66}$ et avoir 
exploré les différents sens possibles de chaque expression. De même, les deux textes opèrent volontiers, contre les arguments franciscains, un retour à la lettre de l'Évangile. Ces rapprochements ne suffisent toutefois pas à conclure de façon certaine.

Comme l'ont relevé D. Trapp et J. Koch, Johannes Hiltalingen a longuement fréquenté les différents avis théologiques de Benoît XII, puisqu'il fait également usage des critiques portées contre maître Eckhart, Michel de Césène et Guillaume d'Ockham ${ }^{67}$. Il devait avoir à sa disposition un recueil de ces textes, conçu sur le même modèle qu'un volume perdu, autrefois conservé dans la bibliothèque pontificale. L'inventaire dressé sous Urbain V (1369) le décrit de la sorte, sous le numéro 382, sans mentionner le nom de l'auteur des avis :

Item magnus liber contra dicti magistri Ekardi, magistri Guillelmi de Ocham, fratris Petris Iohannis Olivi, Ioachim super Apocalipsy et magistrum Michelem de Sezena, coopertus corio rubeo, qui incipit in secundo corundello primi folii : sunt, et finit in ultimo corundello penultimi folii ante articulo[s] Ekardi : in communi sed".

Dans l'inventaire dressé sous Grégoire XI (1375), les mêmes avis, attribués à Jacques Fournier en tant que cardinal, occupent cette fois deux volumes. La description du premier d'entre eux mentionne, à la suite des avis précédents, un rapport rendu à propos de l'opinion de Durand de Saint-Pourçain sur la vision béatifique ${ }^{69}$. La rédaction de nouveaux inventaires était parfois menée à l'occasion d'un reclassement des fonds. Dans le cas présent, on constate qu'un effort a été entrepris pour rassembler deux volumes comportant des avis théologiques de Benoît XII. Le même titre global a été reporté pour désigner chacun des deux codex comme première et seconde partie d'un même ensemble, alors même que le second volume ne contenait vraisemblablement que les seuls traités sur la vision béatifique. Il faut donc reconnaître sous cette description l'actuel cod. Vat. lat. 4006, qui est décrit de façon distincte dans tous les autres inventaires de la bibliothèque pontificale. Le point le plus intéressant à noter pour notre propos est que, dans ces deux descriptions, le nom de Joachim n'apparait pas. À la faveur d'un nouveau reclassement, dans l'inventaire de la bibliothèque que Benoît XIII avait emporté avec lui dans son château de Peñiscola, le recueil des avis de Jacques Fournier s'est retrouvé placé en compagnie d'autres œuvres du même auteur, et notamment de son commentaire sur Matthieu; cette fois, le commentaire de l'Apocalypse de Joachim de Fiore est présenté comme l'une des cibles du cardinal cistercien $^{70}$. En revanche, ce nom disparaît dans la dernière description du même codex, réalisée lors de l'inventaire après décès de Benoît XIII"11.

Les historiens se sont interrogés sur la présence du nom de Joachim dans cette liste qui rassemble la crème des personnages censurés durant le pontificat de Jean XXII. Faut-il penser, avec F. Ehrle, qu'une véritable action autonome contre l'abbé de Fiore aurait été engagée, plus de cent ans après son décès ${ }^{72}$ ? Ou doit-on plutôt juger, avec J. Koch, que les descriptions données dans les inventaires doivent être complétées d'un mot indiquant qu'étaient visés, non pas Joachim en personne, mais ses disciples du XIV siècle $^{73}$ ? La probabilité que la même omission se soit reproduite lors de deux opérations de catalogage distinctes est trop faible pour que l'on puisse retenir cette dernière hypothèse. Il est par ailleurs significatif que le nom de Joachim n'apparaisse pas à chaque fois, comme si la présence de l'abbé dans la liste des auteurs censurés n'était pas évidente. Cette situation correspond assez bien à la forme de l'avis contenu dans le manuscrit d'Avignon qui, pour répondre à une interrogation sur le seul cas d'olivi, étend son investigation à la doctrine de Joachim. Le texte peut donc être aussi bien décrit comme portant sur les deux auteurs ou ne concernant que le premier d'entre 
eux. Une telle extension de l'enquête est si rare qu'elle fournit un argument fort pour associer le document anonyme au rapport perdu de Jacques Fournier signalé par ces inventaires ${ }^{74}$.

Jacques Fournier et les béguins

Une autre piste peut venir étayer cette identification. Comme on l'a noté, une particularité de l'auteur de l'avis est sa connaissance du commentaire sur Daniel de Barthélemy Sicard. L'hypothèse qu'il ait été en contact direct avec les béguins persécutés, après 1318, est renforcé par une formule cinglante, employée dans le dernier article de l'avis pour dénoncer le thème d'une survie de l'Église dans un nombre minime d'élus durant le règne de l'Antéchrist: «Il n'a pas été prédit que le Christ devrait être loué et confessé par une Église pauvre de quatre paysans, ou de douze ou cent béguins, mais dans une grande Église ${ }^{75}$.» Or parmi les maîtres en théologie sur lesquels Jean XXII pouvait compter en 1325, l'évêque de Pamiers était le mieux placé pour parler ainsi des pauvres groupes clandestins qui prétendaient incarner à eux seuls l'Église du troisième âge. Ce que nous allons maintenant prouver.

On sait depuis longtemps que le fameux registre d'inquisition de Jacques Fournier, contenant notamment ses interrogatoires de Montaillou, n'est pas le seul qu'il ait fait confectionner pour garder trace de son activité inquisitoriale dans le diocèse de Pamiers. En effet, un registre décrit dans les anciens inventaires de la bibliothèque pontificale ne correspond pas à la forme de celui qui a été conservé. Les recherches d'Anneliese Maier ont permis de rassembler trois descriptions différentes de ce second registre qui permettent d'apporter une précision de taille. Le plus parlant est de reproduire ces trois descriptions :

Item processus domini Benedicti pape contra hereticos, dum erat episcopus Apamiarum, coopertus corio albo, qui incipit in secundo folio post tabulam errorum : dictus, et finit in penultimo folio : in crimine ${ }^{16}$ (Ur. 661).

Item in volumine signato per CCXXV liber errorum et heresum beguinorum tercii ordinis sancti Francisci " (Gr. 726).

Item liber errorum et heresum beguinorum de tercio ordine sancti Francisci moderni temporis, cop. pelle alba et inc. in $2^{\circ}$ folio post tabulam : francisci et finit in penultimo : in crimine (Av. 509).

Par chance, les descriptions physiques de Ur. 661 et Av. 509 permettent d'associer deux informations qui auraient autrement été déconnectées l'une de l'autre. Le registre d'inquisition perdu de Jacques Fournier, lorsqu'il était évêque de Pamiers (selon la description Ur. 661), concernait ainsi les « erreurs et hérésies des béguins du tiers ordre de saint François» (selon Gr. 726 et Av. 509). Cette découverte n'est qu'une demisuprise. Il était en effet étonnant qu'aucun béguin n'apparaisse dans le registre concernant Montaillou, alors que ces derniers, aux mêmes dates, étaient poursuivis et interrogés dans le diocèse voisin de Mirepoix, notamment à Belpech, un centre actif du mouvement, situé à moins de vingt kilomètres de Pamiers. Jacques Fournier avait luimême été associé à ces procédures, puisque le sermon général condamnant les béguins de Cintegabelle et Belpech fut prononcé en sa présence, à Pamiers, les 4 et 5 juillet 1322, par les inquisiteurs de Toulouse et de Carcassonne et l'évêque de Mirepoix ${ }^{79}$.

Le registre contenant les procédures de Montaillou est décrit, sur sa première page, comme concernant "les erreurs des Vaudois", ce qui ne correspond qu'au premier procès contenu dans ce volume. La formule reprise par les catalogueurs d'Avignon était probablement inscrite de la même façon en tête du second registre. Elle signale donc, au minimum, que la première affaire présente dans ce codex concernait les béguins. Il 
aurait pu s'agir de reporter la sentence de juillet 1322. Mais il n'est pas impossible que ce jugement ait incité Jacques Fournier à rassembler dans le même volume d'autres investigations concernant l'hérésie la plus dangereuse et la plus coriace de l'époque, laissant dans un autre recueil, celui que nous connaissons, les affaires dont les enjeux théologiques étaient moindres.

Un autre élément qui peut être apporté comme indice en faveur d'une attribution de l'avis à l'évêque de Pamiers permet en même temps de suggérer l'importance qu'a eu ce document pour préparer la condamnation finale de la Lectura super Apocalipsim. Dans le récit qu'en fait Bernard Gui, qui est l'unique source relatant le fait, la sentence fut prononcée par Jean XXII en consistoire public le 8 février $1326^{80}$. Exactement deux semaines plus tard, le 22 février, Jacques Fournier recevait du souverain pontife, en raison de ses efforts déployés dans l'extirpation de l'hérésie, l'indulgence plénière habituellement remise aux seuls inquisiteurs ${ }^{81}$. Il est difficile de ne voir qu'une simple coïncidence dans la proximité des deux évènements. L'interprétation la plus légitime que l'on puisse en faire serait que Jean XXII a voulu, par ce geste, féliciter l'évêque de Pamiers, aussi bien pour sa contribution savante dans le processus de condamnation d'Olivi que pour son intense activité inquisitoriale - le cas des béguins étant assurément plus important aux yeux du souverain pontife que la résurgence du catharisme dans les hautes vallées de l'Ariège. De fait, dix jours plus tard, Jacques Fournier était transféré sur le siège épiscopal voisin de Mirepoix, dans un diocèse où les béguins constituaient encore le groupe le plus menaçant.

31 Une fois devenu cardinal, c'est encore à lui qu'est revenu la tâche, en 1333, de traiter en appel l'affaire du noble roussillonnais Adhémar de Mosset, proche de Philippe de Majorque, que le roi Jacques III de Majorque dénonçait comme béguii ${ }^{82}$. L'interrogatoire très fourni auquel le soumet le cardinal Fournier démontre une connaissance aiguë de l'hérésie des béguins et de leur principal source d'inspiration. À bien l'observer, on découvre qu'il s'appuie aussi bien sur une interprétation de la Lectura super Apocalipsim présentant des affinités avec l'avis du manuscrit d'Avignon que sur les déclarations des béguins condamnés à Pamiers en juillet 1322. À défaut d'offrir une démonstration complète, il suffira de donner un exemple frappant. Le septième article demande ainsi à Adhémar s'il a entendu dire que dans le troisième âge de l'Église, l'Esprit saint sera donné en telle quantité que les hommes de ce temps ne pécheront plus, à tel point qu'une belle jeune fille pourra faire le voyage de Rome à Compostelle sans susciter ni commettre le moindre péché83. La première partie de cette phrase exprime les préoccupations théologiques de l'auteur de l'avis au sujet de l'«impeccabilité » du troisième âge, tandis que l'exemple de la jeune fille provient directement de la sentence rendue contre le béguin de Belpech, Bernat de Na Jacma ${ }^{84}$. Cet interrogatoire permet donc d'observer le cardinal Fournier au travail, croisant habilement les deux sources dont il disposait dans ses archives ${ }^{85}$.

L'ensemble de ces indices ne laisse aucune place au doute quant à l'auteur de l'avis. Dans sa forme, ce texte correspond à la description d'un écrit perdu de Jacques Fournier. Les citations qu'en fait Johannes Hiltalingen n'apportent pas de confirmation directe, mais elles montrent du moins une similitude formelle dans l'examen doctrinal de différents extraits de la Lectura. Mais, sur le fond, c'est la mise en rapport avec la persécution des béguins qui apporte l'élément le plus significatif. Bien qu'ils ne soient explicitement cités qu'une seule fois dans le texte, ces groupes sont assurément présents à l'esprit de l'auteur. Le cœur de sa démarche vise à déployer toutes les 
conséquences du commentaire d'olivi, afin de montrer que l'avènement d'un troisième âge implique nécessairement une rupture avec l'Église romaine et l'abolition des sacrements, dans des termes qui correspondent effectivement à l'évolution doctrinale des béguins et de certains Spirituels après 1318. Dans ses Allegationes, Bonagrazia de Bergame n'hésitait pas à présenter cette hérésie comme la plus dangereuse que l'Église ait eu à affronter ${ }^{86}$. Au vu des longs extraits de son intervention dans ce dossier, Jacques Fournier ne semble pas loin d'avoir partagé cette opinion. Si la répression de ces groupes a pu occuper une part plus importante de son activité d'inquisiteur qu'on ne le soupçonnait, la réfutation des fondements oliviens de la « subversion » de l'Église dont ils étaient porteurs a bien été l'une des grandes affaires intellectuelles des années 1320 .

\section{NOTES}

1.J. DUVERNOY éd., Le Registre d'Inquisition de Jacques Fournier, évêque de Pamiers (1318-1325), Toulouse, 1965, 3 vols. La bibliographie suscitée par ce document est surabondante.

2.W. J. COURTENAY, « Reflections on Vat. lat. 1086 and Prosper of Reggio Emilia, O.E.S.A », dans C. SCHABEL dir., Theological Quodlibeta in the Middle Ages. The Fourteenth Century, Leyde, 2007, p. 354-355, identifie des questions qui pourraient avoir été disputées à Paris par Fournier, bachelier en théologie, vers 1310-1314. Voir aussi R. E. LERNER, «A Note on the University Career of Jacques Fournier, O. Cist, later Pope Benedict XII », Analecta cisterciensia, 30, 1974, p. 66-69.

3.Benedicti papae Undecimi, In Evangelium D. Matthaei absolutissima commentaria, G. LAZARI éd., Venetiis, apud

D. Zenarium, 1603. Le seul travail consacré à ce texte est A. Maier, « Der Kommentar Benedikts XII. zum Matthaeus-Evangelium » (1968), dans EAD., Ausgehendes Mittealter, t. 3, Rome, 1977, p. 591-600.

4.J. KOCH, « Der Kardinal Jacques Fournier (Benedikt XII) als Gutachter in theologischen Prozessen » (1960), dans ID., Kleine Schriften, Rome, 1973, t. 2, p. 368-386.

5.Seuls les prologues ont été publiés par A. MAIER, « Zwei Proemien Benedikts XII », (1969), dans EAD., Ausgehendes Mittelalter, t. 3, p. 447-479. Voir aussi Ch. TROTTMANN, « Deux interprétations contradictoires de saint Bernard : les sermons de Jean XXII sur la vision béatifique et les traités inédits du cardinal Jacques Fournier ", Mélanges de l'École Française de Rome, Moyen Âge, 105/1, 1993, p. 327-379.

6.Sur l'ensemble des procédures, je me permets de renvoyer en dernier lieu à mon article : «Censures et condamnation de Pierre de Jean Olivi : enquête dans les marges du Vatican ", Mélanges de l'École française de Rome - Moyen Âge, 118/2, 2006, p. 313-373.

7.Sur ces documents, voir E. PÁSZTOR, « Le polemiche sulla Lectura super Apocalypsim di Pietro di Giovanni Olivi fino alla sua condanna », Bulletino dell'Istituto Storico Italiano per il Medioevo, 70, 1958, p. 365-424 ; D. BURR, Olivi's Peaceable Kingdom. A Reading of the Apocalypse Commentary, Philadelphie, 1993, p. 221-239 ; S. PIRON, «Bonagrazia de Bergame, auteur des Allegationes sur les articles extraits par Jean XXII de la Lectura super Apocalipsim d'Olivi, dans Revirescunt chartae, codices, documenta, textus. Miscelleana investigationum medioevalium in honorem Caesaris Cenci OFM collecta, A. CACCIOTTI, P. SELLA éd., Rome, 2002, t. 2, p. 1065-1087.

8.Toutefois, c'est probablement à partir du manuscrit d'Avignon qu'un érudit de Carpentras, Joseph-François Bonnet de Saint-Bonnet, a rédigé dans ses Mémoires historiques une Note sur Pierre-Jean Olive, religieux accusé d'hérésie, Carpentras, Bibl. Mun., 1211, p. 232-235 (je n'ai pas consulté ce manuscrit). 
9.Avignon, BM 1087, fol. 220-275v, décrit dans Catalogue général des manuscrits des bibliothèques publiques de France, Départements, t. XXVII, Avignon, par L.-H. Labande,t. 1, Paris, Plon, 1894, p. 502-504. Je remercie Robert Lerner d'avoir partagé avec moi ses impressions sur ce texte qu'il avait lui-même étudié. Ce que je dirai ici ne va guère au-delà de ce qu'il aurait pu écrire lui-même. Le manuscrit a été examiné sur les microfiches de l'Institut de recherche et d'histoire des textes, d'où l'absence de toute observation codicologique.

10.Catalogue général des manuscrits des bibliothèques publiques de France, Départements, t. XXVII, Avignon, par L.H. Labande, t. 1, Paris, 1894, p. 502-504.

11.Je suis particulièrement reconnaissant à Warren Lewis de m'avoir permis d'utiliser la version révisée de son édition de la Lectura qui doit être prochainement publiée. Je ferai ici référence au premier état de son édition, contenu dans sa thèse de doctorat inédite, Peter John Olivi : Prophet of the Year 2000. Ecclesiology and Eschatology in the Lectura super Apocalipsim, Tübingen, 1972, sous le sigle LSA. L'extrait en question devait débuter aux mots : « Sciendum etiam quod sicut sanctissimus pater noster Franciscus est post Christum et sub Christo primus et principalis fundator et initiator et exemplator sexti status "... (LSA, p. 558). Le manuscrit d'Avignon débute au milieu de la phrase suivante, aux mots : « Deus apparebat et loquebatur Moysi » (LSA, p. 560).

12.Ms. Bibl. Mun. d'Avignon, 1087, fol. 220rb : « In qua vero parte temporis tertii temporis mundi sexti et septimi ecclesie iterum spiritus sanctus infudatur, illa infusione de qua fit mentio in supradictis posicionibus Joachim et Petris Johannis incertum est. »

13.Ibid., fol. 221rb : « Sed cum in hac questione non queratur nisi 'an in tertio tempore huius vite inchoandi in sexto statu ecclesie non solum simplici intelligentia sed et palpativa et gustativa experiencia videatur omnis sapiencia verbi dei incarnati ac potentia deo patris, quia Christus promisit quod cum venit illud spiritus veritatis docebit vos omnem veritatem', solum videndum est omissis primis duobus temporibus mundi et quinque statibus ecclesie, quo tempore incohatum est tertium tempus mundi et sextus status ecclesie, ut videatur si in illo tempore .iii. vel sexto statu ecclesie non solum simplici intelligentia set etiam gustativa et palpativa experiencia videbitur omnis sapiencia verbi dei incarnati et potencia dei patris. »

14.Ibid., fol. 221rb : « et sic secundum eum [=Joachim] iam durasset tertium tempus vel octingentos annos si incepit tempore sancti Benedicti vel per centum .xxxix. annos si incepit tempore quo ipse venit ad Urbanum papam .iii. vel iam sunt .cxxv. anni si incepit secundum eundem mcc. ${ }^{\circ}$ anno ». Voir G. L. POTESTÀ, Il tempo dell'Apocalisse. Vita di Gioacchino da Fiore, Bari, 2004.

15.Ms. Bibl. Mun. d'Avignon 1087, fol. 221va : «Cum fratrem etiam P. Johannis non conveniens cum dicto Joa. dicat quod dictus tercius status inceptus fuit tempore sancti Francisci cuius regula canonizata fuit sub anno domini $\mathrm{m}^{\circ} \mathrm{Cc}^{\circ} \mathrm{Xxiii}{ }^{\circ}$, a quo tempore jam effluxerunt centum et duo anni. »

16.Cf. S. PIRON, «La critique de l'Église chez les Spirituels languedociens », dans L'Anticléricalisme en France méridionale, milieu XII ${ }^{e}$-début XIVe siècle Toulouse, 2003 (Cahiers de Fanjeaux, 38), p. 77-109; voir p. 89 pour la citation de l'extrait utilisé dans le ms. Bibl. Mun. d'Avignon, 1087, fol. 221va-vb.

17.W. H. MAY, « The Confession of Prous Boneta, Heretic and Heresiarch » dans J. H. MUNDY, R. W. EMERY, B. N. NELSON éd., Essays in Medieval Life and Thought Presented in Honor of A. P. Evans New York, 1955, p. 11-12: «Quando scriptura fratris Petri Joannis fuit condempnata [...] de quo tempore erunt duo anni in instanti festo nativitatis Domini nunc vero transacto. » La meilleure présentation d'ensemble de Prous est fournie par L. Burnham, « The Visionary Authority of Na Prous Boneta », dans A. Boureau, S. Piron éd., Pierre de Jean Olivi (1248-1298). Pensée scolastique, dissidence spirituelle et société, Paris, 1999, p. 319-340 et EAD., So Great a Light, So Great a Smoke : The Beguin Heretics of Languedoc, Ithaca (N.Y.), 2008.

18.Ms. Bibl. Mun. d'Avignon, 1087, fol. 221vb-222ra : « Transactum etiam sit tempus quo secundum P. Jo. antichristus verus debuisset complevisse suum cursum cum nunc computetur annus incarnationis domini MCCCXXV, et tamen secundum eum post destructionem babilonie, idest ecclesie carnalis, et ante morte 
antichristi dicta infusio spiritus sancti esse debebat, que tamen ut constat facta non est, cum nec sit destructa ecclesia romana quam ipse ecclesiam carnalem vocat, ymo est in culmine sue dignitatis. »

19.Ibid., fol. 222rb : «Cum igitur isti duo, scilicet Joachim et P. Johannis inter se multum discordant in supradictis, eventus etiam rerum manifeste hostendat eos falsum dixisse ; cum non eveniret illud quod predixerunt in tempore vel circa tempus per eos prefixum, clare est eos non esse prophetas domini sed prophetas erroris. »

20.Ibid., fol. 225ra : «Quod vero dicunt Babiloniam id est carnalem romanam ecclesiam ante mortem antichristi destruendum in fine secundi status et in principio tercii, non solum est falsum sed etiam est blasffemum ac hereticum. » La démonstration court jusqu'au fol. 229va.

21.Ibid., fol. 229va : « Subpositis tamen omnibus supradictis falsis tanquam si vera essent, queritur an catholice possit dici. »

22.Ibid., fol. $229 \mathrm{va}-\mathrm{vb}$ : « Sed prius quia dubium est quid significare voluit adinventor istorum verborum 'non solum simplici intelligentia sed et gustativa et palpativa experiencia', que verba sunt in cognitione intellectuali qua deus cognoscitur, methaforice forte sumpta ex illo quod dicitur in psalmo Gustare et videre quam suavis est dominus, et ex illo loco Jere. $\mathrm{xv}^{\circ}$ Inventi sunt sermones tui et comedi eos, et factum est mihi verbum tuum in gaudium et in leticiam cordis, et $\mathrm{ex}^{\circ} \mathrm{x}^{\circ}$ Factus est sermo domini in corde meo quasi ignis exestuans claususque in ossibus meis et deffeci ferre non sustinens, sic quod in prima auctoritate sermo domini dicitur gustari et in secunda tangi. »

23.Ibid., fol. 230vb : « Tertius intellectus dictorum verborum potest esse quod in dicto tercio statu detur cognitio dei hominibus qua non solum videatur deus in se sed videatur in hominibus, et per ipsosmet qui talem cognitionem habebunt et in aliis hominibus et omnibus aliis creaturis non per creaturas sed in ipsis creaturis. [...] plena et perfecta cognitione humanus intellectus persistat sic quod nulla alia re nisi de deo vel propter deum cogitetur, quasi vilia alia reputando, et quod sic existens in tali cognitione videat omnem veritatem sapiencie verbi dei incarnati et omnem potenciam dei patris, falsum intellectum habarit ac etiam hereticum quia in vita presenti non sic deus videbitur sed in vita futura. »

24.Ibid., fol. 233va : « Unde cum ex ista opinione istorum sequatur necessario, ut videtur, quod homines in vita presenti existentes sint immortales et comprehensores, nulla fide vel sacramentis fidei indigentes, et sine peccato et inpecabiles effecti, quorum quolibet est heresis dampnata in concilio Viennensi per dominum Clementem papam Vm in Sexto libro, de hereticis, Ad nostrum qui desideranter. » Sont plus précisément signalés les premier, quatrième et huitième articles, portant sur l'impeccabilité et l'inutilité des sacrements. Sur cette bulle et son contexte, voir R. E. LERNER, The Heresy of the Free Spirit in the Late Middle Ages, Berkeley, 1972.

25.Ms. Bibl. Mun. d'Avignon 1087, fol. 235vb : « Si vero intelligatur questio iuxta dicta Joachimi, scilicet quod in tertio statu omnium figurarum et obscuritatum sacre scripture tam novi quam veteris testamenti per spiritum sanctum declaratio in mentibus hominum illius status imprimetur, sic quod omnis obscuritatis scripture erunt eis note per spiritum sanctum loquentem in eis et exprimentem ac docentem omnem veritatem, quam expressionem veritatis ipse vocat evangelium eternum ... » Suivent deux longues citations de la Concordia, II, 20 et II, 37 et une citation du prologue de la Lectura super Apocalipsim d'olivi. La réfutation s'étend jusqu'au fol. 239ra.

26.Ibid., fol. 237va-239ra.

27.Ibid., fol. 239ra : « Nec videntur valere illa que aliqui dicunt ad excusandum predicta verba predicti P. Jo. » Comparer avec Bonagrazia, Allegationes, Ms. Paris, BnF lat. 4190, fol. 47r-48r.

28. Ibid., fol. 241va-vb : « Nec solum dicti P. Io. et Ioa. preferunt homines tertii status in scientia apostolis qualitercumque, set etiam quantum ad obiecta et quantum ad modum sciendi quia apostoli imperfecte et in parte sciverunt, isti vero secundum eos scient clare et manifeste omnem veritatem sapiencie verbi dei incarnati et potenciam dei patris quod tamen apostoli non sciverunt. » 
29. Voir plus bas l'examen des renvois croisés entre les différentes parties.

30.Ms. Bibl. Mun. d'Avignon, 1087, fol. 242va : « Cum quedam nova doctrina temporibus istis insurrexerit, ex qua multi in errores diversos sunt prolapsi et timendum est nec plures in posterum ruant, que doctrina per multos doctores in teologia inventa est et adiudicata erronea, temeraria, scismativa, divinatoria ac in multis ecclesie scandalosa, et pauca aut nulla habens utilia.»

31.Ibid., fol. 242vb : « Ponendo primo quemlibet de articulis reputatis suspectos per dictos magistros ac etiam verba dictorum magistrorum, ac deinde illud quod michi de dictis articulis videbitur, ac postea dicere aliquid de excusationibus quas aliqui pretendunt pro verbis dicte postille ut heretica vel erronea non dicantur.»

32.Les citations couvrent les $\S 8-7,17,32-45$ (LSA, édition révisée), alors que le premier article de la Lettre concerne les $§ 25-38$ et le deuxième le début du § 17 .

33.Ms. Bibl. Mun. d'Avignon, 1087, fol. 244rb : « Quod vero postea dicit, sciendum est quare 'sextus status semper describitur ut notabiliter preeminens quinque primis et sicut finis priorum et tanquam initium novi seculi evacuans quoddam vetus seculum, sicut status Christi evacuavit Vetus Testamentum et vetustatem humani generis ; unde et quasi circulariter sic iungitur primo tempori Christi ac si tota ecclesia sit una spera, et ac si in septimo [LSA :sexto] eius statu secundo incipiat status Christi habens sua septem tempora sicut habet totus decursus ecclesie, sic tamen quod septimus status sexti sit idem cum septimo statu totius ecclesie. »

34.Ibid., fol. $244 \mathrm{va}$ : « Sequitur quod sextus status ecclesie notabiliter preemineat non solum statui apostolorum set etiam satui Christi quia Christus fundator ecclesie ac etiam fundamentum non potest excludi a primo statui fundationis ecclesie. »

35.Ibid., fol. 245va : « Et cum postea subdit quod est 'inicium novi seculi evacuans quoddam vetus seculum', clare vult dicere quod in sexti statu, ad minus circa finem dicti status quod ipse vocat septem partem ipsius sexti status, evacuabitur status evangelii vel novi testamenti, sicut evacuatum fuit vetus testamentum et status populi veteris testamenti post passionem Christi. » Les extraits de Joachim proviennent de Concordia, livre 5, dist. 1, ch. 22, 27-28.

36.Ibid., fol. 247va : « Et si hoc modo intelligant fieri renovationem in dicto sexto statu seculi, vel hominum, quod in hominibus nullum fit peccatum per quod homines sunt vetusti vetustate peccati, tunc est error clarus et manifestus Pelagii. »

37.Ibid., fol. 248rb : « Posset intelligi dicta innovatio ecclesie in sexto statu et vetustatis evacuatio quod ecclesia reduceretur ad statum in quo nullus de ecclesia aliquid haberet in proprio vel in communi nisi solum modo simplicem usum facti. »

38.Bonagrazia, Allegationes, fol. 44r, qui renvoie également à Quia quorumdam mentes.

39.Ms. Bibl. Mun. d'Avignon, 1087, fol. 248rb : « Hoc autem divinare est cum ex nulla scriptura hoc possit haberi »; fol. 248vb : «Et sic, cum non probetur ex scriptura quod ecclesie ultimo tempore reduci debeat ad statum in quo fuit tempore apostolorum quantum ad numerum personarum nec quantum ad nichil omnino habere in speciali vel communi vel quantum ad habere solum in communi, presumptuosum est dicere quod ecclesia ad talem statum reduci debeat. »

40.Ibid., fol. 249rb : « Non est etiam convenienter dictum quod propter unam modicam partem populi christiani que beatum Franciscum in abdicatione proprietatis sequta est, status populus christiani sit sic mutatus quod de linea recta flexus fuerit ad lineam circularem et quod omnes illi qui professionem secundum regulam beati Francisci votum faciunt paupertatis sint magis Christo conformes quam martires "; fol. 249va : "Quelibet religio aliquo modo Christo assimilatur et tamen nulla est que in omnibus assimiletur.» 41.Ibid., fol. 250vb : « Vita enim evangelica cum a fundatione ecclesie usque ad finem in aliquibus fidelibus perseveret [...] non fuit inveterata ut renovatione indigeret ... » 
42.Ibid., fol. 250vb-251ra : « Dicere etiam quod in sexto statu renovata est evangelica vita vel solum vel maxime, est blasfemum ac etiam hereticum. [...] nec aliquis ita inveniretur amens qui audet dicere quod quicumque esset professor regule sancti Francisci magis esset imitator Christi et in eo magis esset vita Christi reformata quam in sanctis Laurencio, Vincencio, Martino, Nicholao, Benedicto, Antonio et in aliis viris qui fuerunt homines maxime sanctitatis. »

43.Ibid., fol. 251ra : « Renovationem autem in modo vivendi secundum vitam Christi non posuit apostolus in expropriatione omnis dominii rei temporalis vel in aliis votis religiosorum principialiter consistere, set in iusticia et sanctitate veritatis et carentia viciorum secundum quod deducit ad Effe. .iiii. (Eph. 4,22). »

44.Ibid., fol. 251va : «Cum ergo principaliter homo renovetur in vita Christi per vitationem peccatorum et opera virtutum principalium magis quam per vota [...] non est dicendum quod vita Christi et evvangelica per eum [sc. Franciscum] et professores regule sue fuerit renovata, cum multi alii ante eum et post eum fuerunt in vita Christi renovati, habentes in se ipsis virtutes que ad renovationem hominis in Christo et secundum Christum requiritur. »

45.Ibid., fol. 252ra : « Et quod addunt quod iste sextus status est 'iterate reedificacionis ecclesie simul prime' [...] Nam ecclesia Christi nunquam cadet usque ad finem seculi. »

46.Ibid., fol. 252vb : « Nulla etiam scripture autentica invenitur que dicat quod ecclesia in sexto statu vel tempore antichristi aut post ipsum debeat reduci ad tantam paucitatem personarum sicut fuit in ecclesia primitiva Christi. »

47.Ibid., fol. 254vb : « Quod etiam addunt quod 'prefatus angelus, idest sanctus Franciscus Christi signo signatus per suos signabit futuram miliciam Christi', temerarium est omnino ac divinatorium et nullam rationem habens, quia tunc omnes electi qui contra antichristum pugnaturi sunt per homines ordinis sancti Francisci ad fidem et iusticiam instruerentur et non per alios post destructionem ecclesie carnalis ante pugnam ecclesie contra antichristum.»

48. Ibid., fol. 255ra : «Et quod aliqui dicunt ad excusandum dictum fratris Petrum Jo. quod ipse non ponit duas ecclesias successive set solum unam universalem ecclesiam seu romanam, sicut et unam fidem... »; fol. $258 \mathrm{ra}$ : « Et quod dicunt quod ecclesiam vocat carnalem non quidem romanam vel catolicam set catervam vel ecclesiam reproborum, que vicia et peccata carnalia prosequitur ...»

49.Ibid., fol. 259va : «Et quia perfectionem sexti et septimi status Ecclesie super .v. primos status eius, de qua perfectione dictum est in primo articulo, magis insistit probare per rationes in septimum notabile per totum, idcirco ut materia continuetur et illa que supradicta sunt magis manifestentur visa insufficientia rationum et dictorum eius, in quibus etiam multa erronea ponit ut videbitur infra, et quia iam secundus et tertius articuli quasi totaliter sunt hostensi erronei, idcirco de quarto articulo per magistros condempnato est nunc agendum, et ut videntur ad plenum intencione sua totum septimum notabile de verbo ad verbum est ponendum quod tale est. »

50.Ibid., fol. 269vb : « Ex mistica teologia voluerunt trahere argumentum que tamen argumentabilis non est [...] volunt adaptare quasi omnes sex sacre scripture ad sextum tempus ecclesie quem finxerunt, cum non possent invenire in scriptura claras probationes ad illud quod ponere de suo capite intendebant. »

51.Ibid., fol. 242rb : «Et hoc sufficiant de ista questione quo ad presens. »

52.Ibid., fol. 236rb : «Predicta autem omnia quam sint absurda et erronea tam ex multis que supra dicta sunt in presenti et precedenti questione quam etiam ex illis que dicentur. »

53.D. BURR, Olivi's Peaceable Kingdom, p. 223-224, donne en traduction anglaise la forme que devait revêtir les quatre questions identifiées jusqu'ici.

54.Ibid., fol. 247va : « Nunquam enim homines puri beata Maria excepta fuerunt, sunt vel erunt sine ullo peccato omnino et sine defectu perfecte iusticie, ut etiam satis deductum fuit in .ii. questione »; fol. 255vb : « sicut patet ex prima et secunda questionibus et hiis etiam que dicta sunt circa articulum istum. » 
55.Ibid., fol. 265vb : « et inductum fuit in articulo precedenti »; ID., fol. 266rb : « Sed quia de hac materia multa dicta sunt tam in secunda questione quam in precedenti articulo, non multum insisto ».

56.Ibid., fol. 251va : « Et tamen, ut hostensum fuit in prima questione ius et dominium in aliquibus rebus temporalibus habuit Paulus. »

57.Cf. D. BURR, Olivi's Peaceable Kingdom, p. 224.

58. Le fait est annoncé dans l'introduction, mais les passages correspondants n'ont pas été conservés, cf. Allegationes, fol. 40r : "Secundo ostendam quos articulos reputo hereticos et quare reputo eos hereticos. Et premictam illos quos vestra sanctitas extraxit de postilla, quam frater Petrus Johannis composuit super Apocalipsim. Postea ponam aliquos articulos qui per duodecim magistros in sacra pagina, quibus sanctitas vestra commisit, sunt heretici reputati. »

59.P. NOLD, « Bertrand de la Tour, Omin. Life and Works », Archivum Franciscanum Historicum, 94, 2001, p. 280-281. Le document perdu s'intitulait : Contra quaedam quae videntur excusare ab omni haeresi doctrinam Petri Iohannis.

60.J. KOCH, « Der Kardinal Jacques Fournier » (cité n. 4).

61.D. TRAPP, « Hiltalinger's Augustinian Quotations », Augustiniana, 4, 1954, p. 412-449

62. Ms. Fribourg, Cordeliers, 26, fol. 42rb : « ... prout dominus Benedictus in reprobacione primi articuli Petri Io. capitulo tertio, primo quia licet res proprias in speciali episcopo habere [...] secundo quia inter omnia posita a Paulo ad Thy. 3 nec alibi in sacra scriptura et precipue novi testamenti reperitur pro habitu quod episcopi non propria possint habere ; tertio quia Christus non petiit a Petro cum ipsum vicarium suum constituit ‘vis sine proprio vivere ?' sed 'veni, sequere me’ Math. 4 [rectius 19] et 'Simon Iohannis, diligis me plus his?' Ioh. Ultimo. » Je dois à la générosité de Robert Lerner la communication d'une reproduction de ce manuscrit.

63.Ibid., fol. $42 \mathrm{va}$ : « Secundum corrolarium, si pontificatus Christi stirpi vite ewangelice et apostolice in Petro et apostolis datus fuisset dominio in proprio et communi per paupertatem mundatus, non videtur sequi formaliter quod supremus pontifex ecclesie [...] foret nunc ad hoc obligatus. ». Hiltalingen rappelle un peu plus loin l'origine de cette formulation : « Pro quibus sciendum quod Petrus ille in questione una postille sue super Apocalipsim quesivit utrum catholice dici possit quod pontificatus Christi fuit primo stirpi vite ewangelice et apostolice in Petro et apostolis datus ac deinde utiliter ac rationabiliter ad statum habentem temporalia commutatus. »

64.Ibid. : «Quia apostoli nec in communi nec in proprio ut videtur aliqua bona notabilia habuerunt [...] Ex hiis apparet quod modicum vel nihil concludit totus processus Benedicti 12 contra Petrum Io. articulo primo $\mathrm{ca}^{\circ} 13$ vel ultimus quamvis suppositum Petrum sit hereticum ab ecclesia dampnatum, ut patet idem Benedictus eodem articulo capitulo »; fol. 42vb : "Constat in pontificem romanum multa tenere facere et servare quod Christus non fecit. »

65.Le passage est publié par J. $\mathrm{KOCH}$, op cit., p. 446. Je reproduis ici le troisième point, pour compléter la transcription de J. KOCH qui omet une intéressante allusion à Exiit qui seminat et Exivi de paradiso (passage souligné) : « Tertio modo potest dici vita evangelica status eorum qui dicunt se omnia abdicasse in proprio et communi, sicut de se asserunt fratres minores aliqui, ut satis notum, et in isto sensu videtur quod predictus Petrus qui fuit minor, moverit antedictam questionem, et sic sumendo questionem tunc fundatur questio in suo supposito in Clementis 5 et Nicolay $4^{\circ}\left[\right.$ rectius : $\left.3^{\circ}\right]$, et hunc verba eorum Extra, de verborum significatione, et sub isto sensu suppositum questionis dicit dominus Benedictus hereticum fore. »

66. Voir par exemple, J. KOCH dir., op. cit., p. 445 : « Hos sensus prosequitur $10^{\circ}$ capitulo ibidem et tunc tractat secundum membrum questionis, scilicet qualiter fuerit commutatus, scilicet essentialiter vel quoad modum. »

67.D. TRAPP, « Augustinian Theology of the Fourteenth Century », Augustiniana, 6, 1956, p. 244, qui relève 36 mentions de Benoît XII et 15 de Jean XXII. 
68.F. EHRLE, Historia bibliothecae romanorum pontificium, tum Bonifatianae tum Avenionensis, Rome, 1890, p. 316. 69.Ibid., p. 499 : «Item, in volumine signato per CLV, dicta et responsiones fratris Iacobi tituli sancte Prisce presbyteri cardinalis ad articulos datos per dominum Iohannem papam xxii ex dictis fratris Ekardi, Michaelis, Guillelmi de Ocham et Petri Iohannis ordinis fratrum minorum <et> de animabus sanctorum exutis de corpore an videant deum ante diem iudicii, secunda pars » (Gr. 655) ; le « et » est suppléé par A. MAIER, «Zwei prooemien Benedikts XII », p. 457, afin de distinguer l'avis sur Durand de Saint-Pourçain de l'avis contre Olivi, qui étaient confondus par Jean-Marie VIDAL, « Notice sur les œuvres du pape Benoît XII », Revue d'histoire ecclesiastique, 1905, p. 563-564 ; «Item, in volumine signato per CLVI, prima pars contra articulos magistrorum Ekardi, Michaelis, Guillelmi de Ocham et Petri Iohannis » (Gr. 656).

70.M. FAUCON, La librairie des papes d'Avignon (1316-1420), Paris, 1886-1887, t. 2, p. 49 : Item responsiones ejusdem domini Benedicti contra dicta magistri Eckardi, magistri Guillermi de Ocham, fratris Petri Joannis, abbatis Joachim super Apochalipsim, et magistri Michaelis de Sezena (Pa 98).

71. M-H. JULLIEN de POMMEROL et Jacques MONFRIN, La Bibliothèque pontificale à Avignon et à Peñiscola pendant le grand schisme d'Occident et sa dispersion. Inventaires et concordances, I, Rome, 1991 (Coll. de l'EFR, 141), p. 386 : «Item alius liber scriptus in pergameno copertus de corio rubeo continens tractatum contra dicta magistri Equardi et Guillelmi de Ocam et contra dicta fratris Petri Johannis Olivi ordinis minorum et contra dicta Micaelis de Sazena, qui incipit in primo colondello secundi folii // scriberem et finit in eodem dictum Micha // $(\mathrm{Pb} 93)$ » ; le volume fut donné à un curé de la province de Valence, grossoyeur des lettres pontificales : «Fuit traditus Guillelmo de Cardona pro sua provisione. »

72.F. EHRLE, Der Sentenzenkommentar Peters von Candia, des Pisaner Papstes Alexanders V, Münster, 1925, p. 87. C. Schmitt, Un Pape réformateur et un défenseur de l'unité de l'Église, Benoît XII et l'Ordre des Frères mineurs : 1334-1342, Quaracchi, 1959, p. 161, conjecture que les deux auteurs ont été incriminés au titre de la pauvreté évangélique.

73.J. KOCH, op. cit., p. 369. Cf. A. MAIER, Zwei Prooemien Benedikts XII, op. cit., p. 458, n. 34.

74. Seul Guido Terreni, dans sa Summa de haeresibus, plus tardive, effectue le même rapprochement.

75.Ms. Bibl. Mun. d'Avignon, 1087, fol. 266ra : « Non enim Christus predictus est esse laudandus et confitendus in ecclesia parva quatuor rusticorum vel duodecim aut centum beguinorum, set in ecclesia magna ...»

76.F. EHRLE, op.cit., p. 338.

77.Ibid., p. 503

78.A. MAIER, « Der Katalog der päpstlichen Bibliothek in Avignon vom Jahr 1441 » (1963), Ausgehendes Mittelalters, t. 3, p. 144

79. Cf. Le Livre des sentences de l'inquisiteur Bernard Gui, 1308-1323, ed. A. PALES-GOBILLIARD, Paris, 2002, p 1276-1426. Le jugement a été rendu à Pamiers afin de centraliser la condamnation d'un réseau actif dans les juridictions des inquisiteurs de Carcassonne et de Toulouse.

80.Bernard Gui relate deux fois cette sentence, dans le Cathalogus brevis ... de romanis pontificibus et dans les Flores Chronicarum. Cf. E. BALUZE, Vita paparum Avinionensium, ed. G. MOLLAT, Paris, 1916, t. 1, p. 142 et 166. 81.Jean XXII, Lettres communes, éd. G. MOLLAT, $n^{\circ}$ 24466: « Eidem qui in inquisitione haereticae pravitatis multos pertulit labores, indulgetur illa plena suorum peccatorum venia quae inquisitoribus pravitatis ejusdem per privilegia S. A. apostolica est concessa, dum pro hujusmodi inquisitionis negotio laboraverit. » 82.J. M. VIDAL, « Procès d'inquisition contre Adhémar de Mosset, noble roussillonnais, inculpé de béguinisme (1332-1334) », Revue d'histoire de l'Eglise de France, 1, 1910, p. 559-589, 682-699, 711-724.

83.Ibid., p. 579 : « Interrogatus si audivit ... quod illo tempore quod sic habundanter dabitur Spiritus sanctus hominibus tertii status, quod dicti homines non peccabunt, nec peccandi habebunt propositum, et in tantum quod si una pulcra puella tunc iret de Roma usque ad Sanctum Jacobum de Galicia, quod non inveniret aliquem qui eam sollicitaret ad peccatum, nec ipsa ad peccatum sollicitaretur. » 
84. Le Livre des Sentences, p. 1334 : «Item dixit se credidisse quod post mortem antichristi totus mundus erit fidelis et benignus, et in tantum quod una puella virgo poterit sola ire de Roma usque ad Sanctum Jacobum et non inveniet qui eam ad malum sollicitet. » L'édition d'A. Palès Gobilliard, dépourvue de toute annotation critique, ne signale ni les sources, ni les remplois des dépositions des béguins.

85. Un relevé complet des sources mises en œuvre dans l'interrogatoire d'Adhémar de Mosset permettrait de faire apparaittre les nuances particulières de la lecture par Jacques Fournier de cette hérésie, et notamment son interprétation sociale de la subversion des pauvres béguins.

86. Allegationes, fol. 40r: « Multo periculosior et magis subversiva totius status ecclesiastici et specialiter romane ecclesie est ista secta fundata omnino in ista postilla quam secta pauperum de Lugduno aut Donatistarum seu Valdensium hereticorum. »

\section{RÉSUMÉS}

Le manuscrit 1087 d'Avignon contient des fragments d'un long avis rendu en 1325 à Jean XXII, préparatoire à la condamnation du commentaire de l'Apocalypse de Pierre de Jean Olivi. Ce texte, qui englobe également dans sa critique les oeuvres authentiques de Joachim de Fiore, correspond à un écrit perdu de Jacques Fournier, autrefois conservé à la bibliothèque pontificale d'Avignon. Un autre volume perdu, comparable au célèbre registre d'inquisition de Jacques Fournier, contenait les enquêtes menées dans les mêmes années par l'évêque de Pamiers contre les béguins de son diocèse. La lutte, doctrinale et inquisitoriale, contre cette hérésie a donc constitué un volet important des activités du futur pape Benoît XII.

A lost tract of Jacques Fournier re-discovered. The codex Avignon 1087 contains fragments of a long advice, submitted to John XXII in 1325 as part of the trial against Peter John Olivi's Lectura super Apocalipsim, that was finally condemned the following year. Interestingly, this document also includes a criticism of Joachim of Fiore. It is shown that this text corresponds to a lost tract of Jacques Fournier, formerly kept in the Avignon pontifical library. This ascription can be confirmed through the author's knowledge of the Languedoc Béguins. Fournier had prepared for himself a copy of their depositions in a lost inquisition register, and made use of them in another 1333 trial. The fight, both doctrinal and inquisitorial, against this heresy appears thus to have been one of the main activities of the future pope Benedict XII, and a cause of his quick ascent in Avignon in the decade before his election.

\section{INDEX}

Keywords : béguins, Benedict XII, heresy, inquisition, Jacques Fournier, Joachim of Fiore, Peter John Olivi, Revelation (Book of)

Mots-clés : Apocalypse, Benoît XII, hérésie, Joachim de Fiore, Pierre de Jean Olivi 
AUTEUR

\section{SYLVAIN PIRON}

Groupe d'Anthropologie Scolastique, CRH, École des Hautes Études en Sciences Sociales, 54 boulevard Raspail, 75006 Paris 